\title{
Hearing Improvement after Bevacizumab in Patients with Neurofibromatosis Type 2
}

\section{Citation}

Plotkin, Scott R., Anat O. Stemmer-Rachamimov, Fred G. Barker, Chris Halpin, Timothy P. Padera, Alex Tyrrell, A. Gregory Sorensen, Rakesh K. Jain, and Emmanuelle di Tomaso. 2009. "Hearing Improvement after Bevacizumab in Patients with Neurofibromatosis Type 2." New England Journal of Medicine 361 (4) (July 23): 358-367. doi:10.1056/nejmoa0902579.

\section{Published Version}

10.1056/nejmoa0902579

\section{Permanent link}

http://nrs.harvard.edu/urn-3:HUL.InstRepos:32631201

\section{Terms of Use}

This article was downloaded from Harvard University's DASH repository, and is made available under the terms and conditions applicable to Other Posted Material, as set forth at http:// nrs.harvard.edu/urn-3:HUL.InstRepos:dash.current.terms-of-use\#LAA

\section{Share Your Story}

The Harvard community has made this article openly available.

Please share how this access benefits you. Submit a story.

\section{Accessibility}




\title{
Hearing Improvement after Bevacizumab in Patients with Neurofibromatosis Type 2
}

\author{
Scott R. Plotkin, M.D., Ph.D., Anat O. Stemmer-Rachamimov, M.D., \\ Fred G. Barker II, M.D., Chris Halpin, Ph.D., Timothy P. Padera, Ph.D., \\ Alex Tyrrell, Ph.D., A. Gregory Sorensen, M.D., Rakesh K. Jain, Ph.D., \\ and Emmanuelle di Tomaso, Ph.D.
}

\begin{abstract}
From the Departments of Neurology (S.R.P.), Pathology (A.O.S.-R.), and Radiation Oncology (T.P.P., A.T., R.K.J., E.T.); the Cancer Center (S.R.P.); the Neurosurgical Service (F.G.B.); and the A.A. Martinos Center for Biomedical Imaging (A.G.S.) - all at Massachusetts General Hospital; and the Department of Audiology, Massachusetts Eye and Ear Infirmary (C.H.) - all in Boston. Address reprint requests to Dr. Plotkin at Yawkey $9 \mathrm{E}$, Massachusetts General Hospital, 55 Fruit St., Boston, MA 02114, or at splotkin@ partners.org.
\end{abstract}

This article (10.1056/NEJMoa0902579) was published on July 8,2009 , at NEJM.org.

N EnglJ Med 2009;361:358-67.

Copyright (c) 2009 Massachusetts Medical Society.

\section{A BSTRACT}

\section{BACKGROUND}

Profound hearing loss is a serious complication of neurofibromatosis type 2, a genetic condition associated with bilateral vestibular schwannomas, benign tumors that arise from the eighth cranial nerve. There is no medical treatment for such tumors.

\section{METHODS}

We determined the expression pattern of vascular endothelial growth factor (VEGF) and three of its receptors, VEGFR-2, neuropilin-1, and neuropilin-2, in paraffinembedded samples from 21 vestibular schwannomas associated with neurofibromatosis type 2 and from 22 sporadic schwannomas. Ten consecutive patients with neurofibromatosis type 2 and progressive vestibular schwannomas who were not candidates for standard treatment were treated with bevacizumab, an anti-VEGF monoclonal antibody. An imaging response was defined as a decrease of at least $20 \%$ in tumor volume, as compared with baseline. A hearing response was defined as a significant increase in the word-recognition score, as compared with baseline.

\section{RESULTS}

VEGF was expressed in 100\% of vestibular schwannomas and VEGFR-2 in $32 \%$ of tumor vessels on immunohistochemical analysis. Before treatment, the median annual volumetric growth rate for 10 index tumors was $62 \%$. After bevacizumab treatment in the 10 patients, tumors shrank in 9 patients, and 6 patients had an imaging response, which was maintained in 4 patients during 11 to 16 months of follow-up. The median best response to treatment was a volumetric reduction of $26 \%$. Three patients were not eligible for a hearing response; of the remaining seven patients, four had a hearing response, two had stable hearing, and one had progressive hearing loss. There were 21 adverse events of grade 1 or 2 .

\section{CONCLUSIONS}

VEGF blockade with bevacizumab improved hearing in some, but not all, patients with neurofibromatosis type 2 and was associated with a reduction in the volume of most growing vestibular schwannomas. 
$\mathrm{N}$ EUROFIBROMATOSIS TYPE 2 IS A DOMInantly inherited genetic condition with a birth prevalence of 1 in $25,000 .{ }^{1}$ Bilateral vestibular schwannomas (also known as acoustic neuromas), which are benign tumors composed of neoplastic Schwann cells that arise from the eighth cranial nerve, are the hallmark of neurofibromatosis 2. These tumors cause progressive hearing loss in most patients with neurofibromatosis type 2, who commonly lose all functional hearing during early adulthood or middle age. Standard therapy for growing sporadic, unilateral vestibular schwannomas includes surgical removal or radiation therapy. Both treatments usually achieve tumor control, but at the frequent cost of hearing loss in the affected ear. ${ }^{2,3}$

Treatment options are limited for patients with neurofibromatosis type 2 and a growing vestibular schwannoma ipsilateral to the only ear with hearing. Results of surgery and radiation therapy for vestibular schwannomas associated with neurofibromatosis type 2 are worse than for sporadic tumors, because rates of tumor control are reduced and iatrogenic hearing loss is more frequent..$^{4-6}$ Although the morbidity of active treatment can be prohibitively high, the consequences of unchecked tumor growth (including progressive brain-stem compression) are also severe. Currently, no medical treatments for tumors associated with neurofibromatosis type 2 are available, and a safe and effective treatment for these patients is needed.

Vascular endothelial growth factor (VEGF) is a critical mediator of tumor angiogenesis and vessel permeability. ${ }^{7-9}$ VEGF and its receptor VEGFR-1 have been detected in schwannomas, and increased levels of these factors correlate with increased rates of tumor growth. ${ }^{10-14}$ Although schwannomas are not considered vascular tumors, the non-VEGF-related, antiangiogenic compound AGM-1470 inhibits angiogenesis and reduces the growth of nerve-sheath tumors in mouse models. ${ }^{15}$ The VEGF-neutralizing antibody bevacizumab has been approved by the Food and Drug Administration for use in the treatment of cancers, but no studies have been performed in patients with neurofibromatosis type 2 or vestibular schwannomas.

Here we describe a retrospective study of 10 consecutive patients with neurofibromatosis type 2 and growing vestibular schwannomas who were treated with bevacizumab. We chose this agent on the basis of tumor immunohistochemical analyses suggesting a potential pathophysiological role for the VEGF pathway in vestibular schwannomas. We provide radiologic and audiologic evidence that bevacizumab treatment offered durable clinical benefit to some patients, including a reduction in tumor volume and an improvement in chronic hearing loss.

\section{METHODS}

\section{IMMUNOHISTOCHEMICAL ANALYSES}

Paraffin-embedded tissue sections from 21 schwannomas associated with neurofibromatosis type 2, 22 sporadic schwannomas, and 9 normal spinalnerve roots were immunostained with the following antibodies: CD31, VEGF, VEGFR-2, plateletderived growth factor receptor $\alpha$ (PDGFR- $\alpha$ ), PDGFR- $\beta$, neuropilin- 1 , neuropilin-2, semaphorin $3 \mathrm{~A}$, and semaphorin 3F. Semiquantitative analysis was performed by two authors, who scored the intensity of tumor-cell and blood-vessel staining on a scale of 0 (no staining) to 3 (strong staining). A customized software analysis tool was used to determine the number of vessels, perimeter, the minor axis of best fitted ellipse (representative of vessel diameter), and the total surface covered by vascular spaces. The same method was used, with the substitution of either VEGFR-2 or neuropilin-2 labeling, to determine the proportion of vessels expressing these VEGF receptors (for details, see the Supplementary Appendix, available with the full text of this article at NEJM.org).

\section{SELECTION OF PATIENTS}

We offered bevacizumab on a compassionate-use basis to patients who fulfilled the clinical diagnostic criteria for neurofibromatosis type $2,{ }^{16}$ had evidence of progressive vestibular schwannomas, and were considered poor candidates for surgery and radiation therapy or declined these treatments. Consecutive patients who met these criteria and had received at least one bevacizumab dose as of August 1, 2008, were included in the analysis.

\section{CLINICAL EVALUATIONS}

The intervals for clinical evaluations were determined before treatment of the first patient. Base- 
line magnetic resonance imaging (MRI) and audiology were performed within 1 month before starting treatment. Clinical evaluation, which included a physical examination, complete blood count, blood chemical analysis, and urinalysis, was performed every 2 to 4 weeks during treatment. Tumor response was monitored with the use of serial MRI scanning at clinical visits at months 1,3 , and 6 and every 3 months thereafter. Tumor volumetric analysis was performed as described previously. ${ }^{17}$ To investigate whether tumor shrinkage might be related, in part, to a decrease in intratumoral vasogenic edema, we subsequently determined the mean apparent diffusion coefficient (a measure of the magnitude of diffusion of water molecules within tissue and a marker of edema on imaging with $\mathrm{MRI}^{18}$ ) within vestibular schwannomas at baseline. Dynamic contrast-enhanced MRI (DCE-MRI) was performed in Patient 6 to evaluate changes in blood flow and vascular permeability after treatment. ${ }^{18}$ Hearing response was monitored with the use of serial audiologic evaluations, including determination of pure-tone thresholds and word-recognition scores (for details, see the Supplementary Appendix). ${ }^{19,20}$ Data regarding toxic effects were collected monthly during routine clinic visits, and adverse events were scored according to the Common Terminology Criteria for Adverse Events, version 3.0.

\section{DEFINITIONS OF IMAGING AND HEARING RESPONSES}

We established definitions of imaging and hearing responses after noting a clinical benefit in the first patient. An imaging response was defined as a decrease of at least $20 \%$ in tumor volume, as compared with the pretreatment baseline. ${ }^{21}$ Volumetric changes of $15 \%$ or $20 \%$ have been used to define response and progression in early-phase trials involving patients with plexiform neurofibromas associated with neurofibromatosis type $1.22,23$

A hearing response was defined as a significant increase in the word-recognition score, as compared with the baseline score. ${ }^{24}$ Patients were eligible for a hearing response if their pretreatment word-recognition score allowed for hearing improvement above the upper limit of the critical-difference threshold (i.e., a wordrecognition score of $94 \%$ or less). Patients with surgically disrupted auditory nerves were not eligible for a hearing response. We used published guidelines ${ }^{25}$ to determine significant differences between word-recognition scores to compare each patient's scores after treatment with baseline values.

\section{STUDY OVERSIGHT}

The institutional review board at Massachusetts General Hospital approved the retrospective chart review and tumor-specimen collections. All patients provided written informed consent for treatment. The authors vouch for the completeness and veracity of the data and data analyses. Bevacizumab was provided as part of clinical care; Genentech, the maker of bevacizumab, had no role in the study.

\section{STATISTICAL ANALYSIS}

For analyses of morphologic features, microvascular density, and measures of diameter and perimeter, we used Bartlett's test for the equality of multiple variances to analyze between-group differences in variance. Analysis-of-variance estimate models were then built and appropriate post hoc testing was performed on the basis of Bartlett's test results. For measures with equal variances, Tukey's test was used. For measures with unequal variances, the Games-Howell test was used. A P value of less than 0.05 was considered to indicate statistical significance.

\section{RESULTS}

\section{IMMUNOHISTOCHEMICAL ANALYSES}

In normal peripheral nerves, the ligands VEGF, semaphorin $3 \mathrm{~A}$, semaphorin $3 \mathrm{~F}$, and the receptors VEGFR-2 and neuropilin-1 were consistently expressed in both Schwann cells and vascular endothelial cells; neuropilin-2 was seen in all Schwann cells but in less than $5 \%$ of blood vessels (Fig. 1A, and Fig. 1 in the Supplementary Appendix). In the schwannomas, tumor cells had expression patterns of VEGF, neuropilin-1, and neuropilin-2 that were similar to those in normal nerve. In contrast, VEGFR-2 was expressed in about half of all schwannomas, and semaphorin $3 \mathrm{~A}$ and semaphorin $3 \mathrm{~F}$ were expressed in only $20 \%$ of schwannomas and at reduced intensity (Fig. 1A and 1B, and Fig. 2 in the Supplementary Appendix). 
VEGFR-2 was found in 32\% of vessels in tumors associated with neurofibromatosis type 2 and in sporadic tumors. In contrast, all vessels expressed neuropilin-1 (similar to normal nerve), and about $10 \%$ of vessels expressed neuropilin-2 (Fig. 1C). Most tumor cells showed little or no expression of PDGFR- $\alpha$ or PDGFR- $\beta$. More than $50 \%$ of tumor specimens expressed both PDGFR- $\alpha$ and PDGFR- $\beta$ on the vascular endothelium, consistent with an angiogenic phenotype (Fig. 3 in the Supplementary Appendix).

Morphometric analysis revealed that schwannomas associated with neurofibromatosis type 2 showed a greater microvascular density (22 vessels per square millimeter) and a larger vessel diameter (mean, $14.2 \mu \mathrm{m}$ ), and perimeter (mean, $91 \mu \mathrm{m}$ ) than normal nerve (with 18 vessels per square millimeter and a mean vessel diameter of $7.9 \mu \mathrm{m}$ and perimeter of $47 \mu \mathrm{m}$ ) (Fig. 1D).

\section{PATIENTS}

The baseline characteristics of the 10 patients in the study are shown in Table 1 . All patients were poor candidates for standard treatment, since eight were at high risk for complete hearing loss and one for bilateral lower cranial-nerve palsies, and one patient had declined surgery or radiation. Patients 1 through 5 had been treated previously with erlotinib chemotherapy ${ }^{26}$ but stopped treatment because of either toxic effects (Patient 1) or tumor growth (Patients 2 through 5).

\section{TREATMENT}

Six men and four women with a median age of 25 years (range, 16 to 53) received intravenous bevacizumab at a dose of $5 \mathrm{mg}$ per kilogram of body weight every 2 weeks. The median annual growth rate in tumor volume before treatment was $62 \%$ (range, 9 to 121). The median duration of treatment was 12 months (range, 3 to 19), and six patients were followed for at least 1 year. Six patients continued to be treated with bevacizumab at the end of the study. Patient 1 stopped treatment after 19 months because of slow growth of a vestibular schwannoma, which caused brainstem compression; the tumor was subsequently resected. Patient 5 discontinued bevacizumab temporarily as she awaited surgical removal of a nonvestibular schwannoma. Patient 7 stopped bevacizumab after 3 months because of progressive growth of a spinal meningioma; he died of complications after surgery to resect the tumor 5 months later. Patient 8 stopped treatment after 8 months because of progressive growth of a vestibular schwannoma and hearing loss.

\section{IMAGING RESPONSE}

Of the 10 tumors, 9 shrank after bevacizumab treatment, and 6 had an imaging response (Table 1, and Fig. 4 in the Supplementary Appendix). The median best response to treatment was $26 \%$ shrinkage (range, $44 \%$ shrinkage to $32 \%$ growth). Of six tumors that had an objective imaging response, the response was maintained in four tumors at the last imaging follow-up 11 to 16 months after the initiation of treatment. Three tumors remained stable (i.e., ranging from a 19\% reduction to a $19 \%$ increase in volumetric growth, as compared with baseline) during bevacizumab treatment, and one patient had an increase in tumor volume of $32 \%$.

A strong correlation was observed between the mean apparent diffusion coefficient at baseline within tumors and the percent decrease in volume at 3 months (Pearson's $\mathrm{r}$ correlation, $-0.87 ; r^{2}=0.75 ; P=0.001$ ) (Fig. 2A). In the single patient with complete DCE-MRI data (Patient 6), a measure of blood flow and vascular permeability decreased by $68 \%$, tumor blood volume decreased by $77 \%$, tumor blood flow decreased by $51 \%$, mean transit time decreased by $9 \%$, and the average vessel size decreased by $70 \%$ at 3 months (Fig. 2B). Twelve months after the initiation of treatment, measures of blood flow and vascular permeability continued to fall. Although the amount of gadolinium enhancement decreased over time, the enhancement pattern within vestibular schwannomas did not change noticeably during treatment (Fig. 2C).

\section{HEARING RESPONSE}

Seven patients were considered eligible for a hearing response. Of the other three patients, two had normal hearing ipsilateral to index vestibular schwannomas at baseline (and thus could not improve), and one had undergone surgical resection of both auditory nerves. A hearing response was observed in four of seven eligible patients (57\%) (Fig. 5 in the Supplementary Appendix). The hearing benefit of bevacizumab was large for Patient 2, whose word-recognition score increased from $8 \%$ to $98 \%$, and for Patient 3, whose 


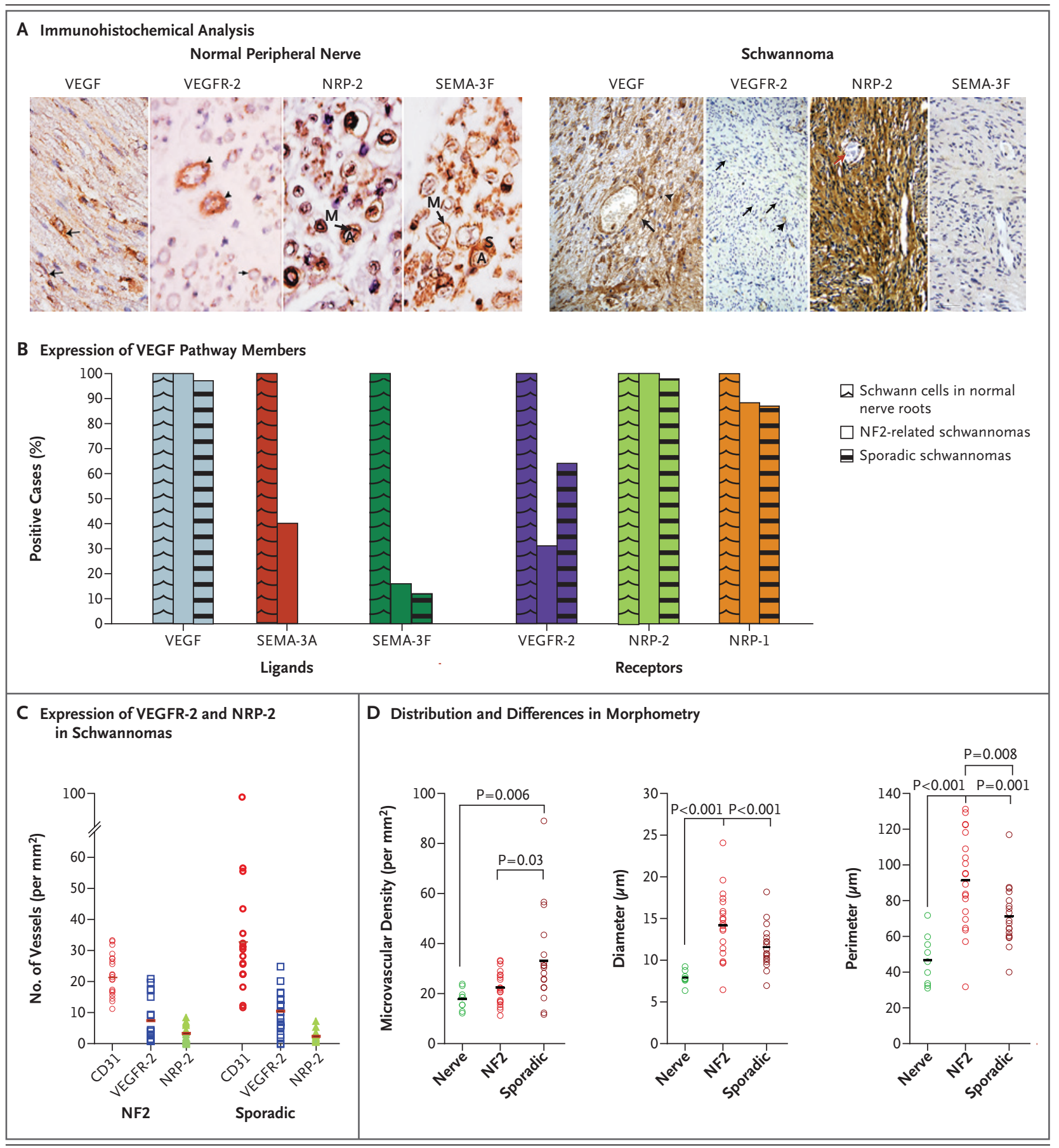

word-recognition score increased from $34 \%$ to $76 \%$ to $94 \%$, also reported improvement in face$76 \%$. Both Patient 2 and Patient 3 regained useful to-face conversation. Patients with a hearing rehearing, and they were able to resume school and sponse showed progressive improvement in word work, respectively. Patient 4 , whose word-recog- recognition, which began about 8 weeks after the nition score increased from $0 \%$ to $40 \%$, reported initiation of treatment and continued to improve better speech-reading (lip-reading), and Patient 5, for as long as 16 months (Fig. 5 in the Supplewhose word-recognition score increased from mentary Appendix). Among the four patients who 
Figure 1 (facing page). Angiogenic Profile of Vestibular Schwannomas.

Panel A shows immunohistochemical analysis of members of the vascular endothelial growth factor (VEGF) pathway in normal peripheral nerve and schwannoma. In normal nerve, staining for VEGF highlights Schwann cells (arrows), and staining for VEGF receptor 2 (VEGFR-2) highlights vessels (arrowheads). Staining for neuropilin-2 (NRP-2) and semaphorin 3F (SEMA-3F) highlights Schwann cells in normal nerve. In schwannomas, VEGF staining highlights tumor cells (arrowhead) and blood vessels (arrow); VEGFR-2 staining highlights some vessels (arrowheads), whereas others remain unstained (arrows). Tumor cells stain diffusely for NRP-2 but not for SEMA-3F. In all panels, brown staining shows positivity for the given antigen, and blue labels the nuclei, with the letter A denoting axon, the letter $\mathrm{M}$ myelin, and the letter S Schwann cell. Panel B shows the percentage of samples with expression of more than 1 on a scale of 0 (no staining) to 3 (strong staining) for the known members of the VEGF pathway (ligands and receptors). SEMA-3F and SEMA-3A are expressed in all nerve roots (3 intensity). In samples from patients with neurofibromatosis type 2 (NF2), $58 \%$ of the tumors have completely negative staining for SEMA-3F, and $22 \%$ are negative for SEMA-3A. In samples from patients with sporadic tumors, $44 \%$ of tumors are negative for SEMA- $3 F$, and $33 \%$ are negative for SEMA-3A. Since none of the samples were scored as 2 or 3 , no bar is shown for SEMA-3A. Panel C shows the quantification of the number of vessels per square millimeter expressing VEGFR-2 and NRP-2 in vestibular schwannomas associated with neurofibromatosis type 2 and sporadic vestibular schwannomas. CD31 was used to count the total number of vessels per square millimeter. There were no significant differences between tumors associated with neurofibromatosis type 2 and sporadic tumors. Panel D shows statistical analysis of the morphometric measures. Diameter, perimeter, and microvascular density were all significantly smaller for the 470 vessels analyzed in normal peripheral nerve roots than in either schwannomas associated with neurofibromatosis type 2 (1215 vessels from 20 patients) or sporadic schwannomas (2104 vessels from 17 patients). The perimeters (or surface area, data not shown) were significantly larger in tumors associated with neurofibromatosis type 2 than in sporadic tumors.

had a hearing response, the result was durable for 11 to 16 months.

Of the three patients who did not have a hearing response, Patient 1 had had no word recognition for at least 8 months before treatment, Patient 8 had a decline in word recognition (44\% to $16 \%$ ) after starting treatment despite initial tumor shrinkage, and Patient 10 had stable hearing despite a sustained reduction in tumor volume. Although Patient 1 did not have a hearing or an imaging response, he had clinical improve- ment that may have been related to treatment. Before treatment, he was receiving hospice care because of intractable headache and vomiting associated with critical brain-stem compression. Within 4 weeks after starting therapy, his headaches and vomiting resolved, and he returned to high school. He remained clinically improved for 19 months of treatment but then required resection of his vestibular schwannoma for tumor growth. Four of five patients reported subjective improvement in baseline tinnitus, and one noted no change.

\section{ADVERSE EFFECTS}

A total of 21 grade 1 or 2 adverse events were reported, including increased levels of aspartate aminotransferase (4 events) and alanine aminotransferase ( 3 events), proteinuria ( 3 events), hypertension ( 2 events), delayed wound healing ( 2 events), hyperkalemia ( 2 events), hyperbilirubinemia (2 events), uterine bleeding (1 event), hypocalcemia (1 event), hypophosphatemia ( 1 event), and hypomagnesemia ( 1 event). Two patients had infected vascular access ports requiring removal. In this small sample of patients, no patients had thromboembolic events, hemorrhage, congestive heart failure, gastrointestinal perforation, or reversible posterior leukoencephalopathy. No patient discontinued treatment because of adverse events, and no grade 3 or 4 adverse events were reported.

\section{DISCUSSION}

Our study showed that bevacizumab treatment was followed by clinically meaningful hearing improvement, tumor-volume reduction, or both in some, but not all, patients with neurofibromatosis type 2 who were at risk for complete hearing loss or brain-stem compression from growing vestibular schwannomas. Our findings confirm that VEGF is produced by schwannoma tumor cells ${ }^{10-14}$ and suggests that this growth factor may participate in tumor growth and hearing loss associated with vestibular schwannomas. The imaging findings indicate that the mean apparent diffusion coefficient at baseline might be a potential marker for volumetric response to antiVEGF therapy. The DCE-MRI data, though limited, further suggest that bevacizumab normalizes the function of tumor vessels and potentially restores a more normal blood-nerve barrier.

These findings have implications for patients 


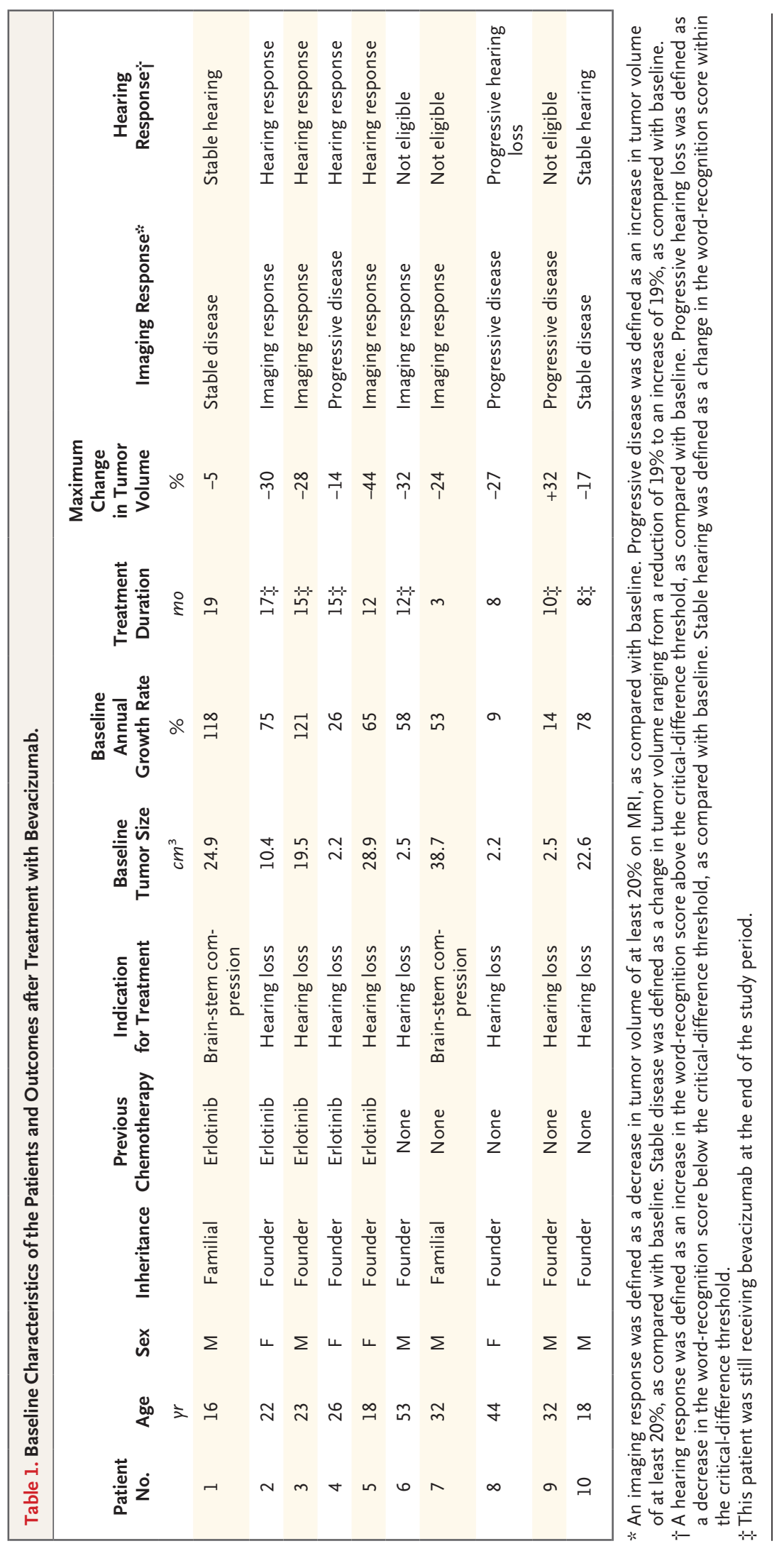


Figure 2. Changes in Magnetic Resonance Imaging (MRI) Measures during Treatment with Bevacizumab. Panel A shows the correlation between mean apparent diffusion coefficients (ADCs) at baseline and subsequent changes in tumor volume for the 10 patients after 3 months of treatment with bevacizumab. Panel B shows dynamic contrast-enhanced MRIs of Patient 6 , with measures of blood flow and vascular permeability indicating the permeability of vestibular schwannomas (with the most permeable areas shown in white and the least permeable areas in dark red). At baseline, permeability is high for both tumors but decreases progressively during 12 months of treatment, as shown by the values beneath the images. Panel $C$ shows representative cranial $T_{1}$-weighted MRI scans of Patient 2 after the administration of contrast material at baseline (left) and after 9 months of treatment (right). $\mathrm{T}_{2}$-weighted scans are also shown at the same time points. The overall decrease in tumor volume $(30 \%)$ can be seen by comparing the tumor outline at baseline (yellow line) with the outline after 3 months of treatment with bevacizumab (red line). The enhancement pattern in vestibular schwannomas did not change noticeably during treatment with bevacizumab.

with neurofibromatosis type 2 and for the study of vestibular schwannoma biology. First, the reduced expression of VEGFR-2 on tumor vessels suggested that interfering with the function of VEGF receptors might be less effective than targeting VEGF directly, which prompted our choice of bevacizumab for treatment. Our data suggest that the current understanding of VEGF-mediated angiogenesis - that VEGF is secreted by tumor cells and then binds to and activates VEGFR-2 on endothelial cells - may be more complex in schwannomas, in which vessels express different VEGF receptors. Further work is needed to understand the role of the semaphorin-neuropilin axis in these tumors.

Second, VEGF inhibition can lead to modest but relevant decreases in the volume of vestibular schwannomas. This effect is notable, given the lack of sensitivity of benign nervous-system tumors to standard cytotoxic chemotherapy. Third, in some patients, VEGF inhibition can lead to a relatively rapid ( $<12$ weeks) improvement in hearing, as reflected by word-recognition scores, with sustained improvement for up to 16 months. Observational studies have shown that word-recognition scores deteriorate over time in patients with vestibular schwannomas and that significant improvement is not expected. ${ }^{27}$ We posit
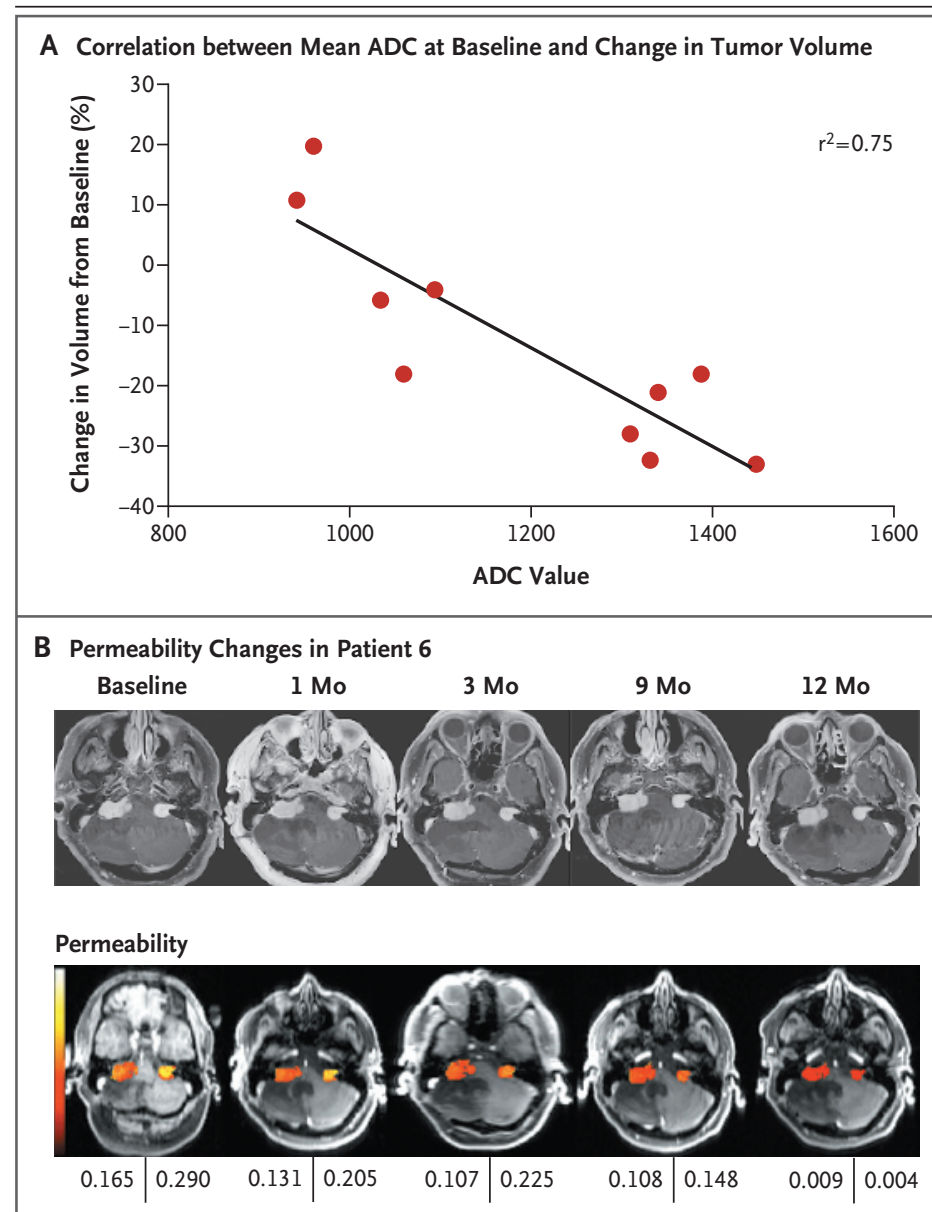

C Imaging Response in Patient 2
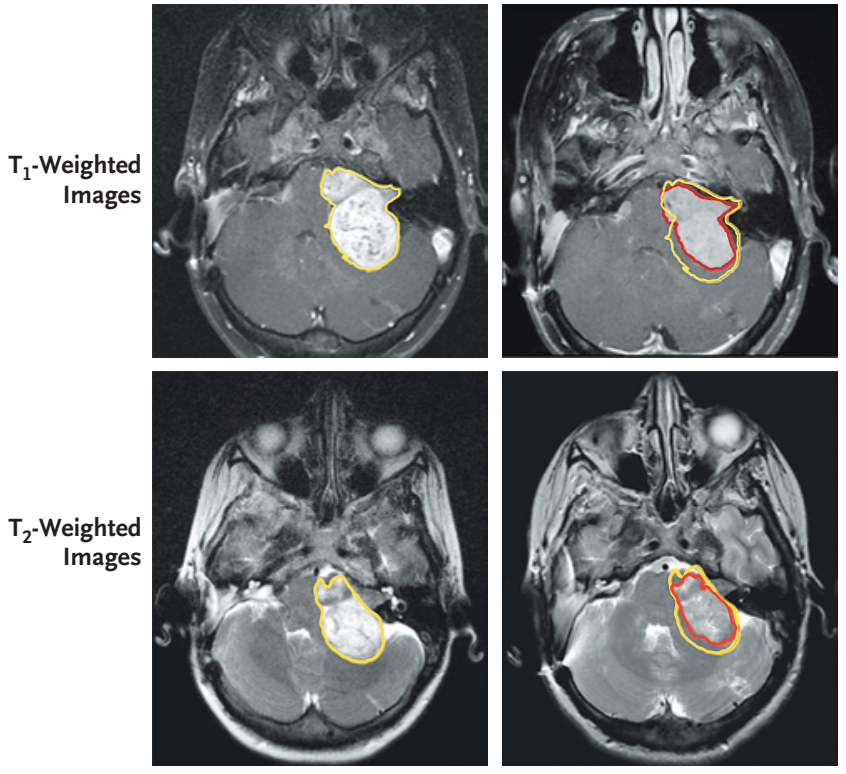
that improvement is due to a reduction in intraneural edema, as well as tumor shrinkage. This assertion is supported by the time course of hearing improvement, the correlation between the mean apparent diffusion coefficient and tumor shrinkage, and the changes in intratumoral vascular permeability, as seen on DCE-MRI. This mechanism is consistent with the vascularnormalization hypothesis ${ }^{28}$ and previous studies of anti-VEGF therapy in malignant brain tumors. ${ }^{18,29}$

To date, four of our six patients with an imaging response and all four patients with a hearing response have continued to respond to therapy for 11 to 16 months. This suggests that response to anti-VEGF therapy can be durable for progressive vestibular schwannomas associated with neurofibromatosis type 2 . In contrast, the median time to progression for recurrent glioblastoma treated with antiangiogenic therapy is about 16 weeks. ${ }^{18,30}$ Patients with benign tumors who benefit from anti-VEGF therapy present unique clinical challenges: they may have prolonged survival during which unanticipated delayed toxic effects could become manifest, and the implications of stopping therapy are unknown, with the potential for rebound tumor growth when monoclonal treatment is stopped. ${ }^{18}$

Although the need for an effective medical therapy for patients with neurofibromatosis type 2 is clear, the risk of toxic effects deserves careful consideration. In our preliminary experience, toxic effects were limited to grade 1 or 2 adverse events during follow-up of up to 16 months, which is not long in comparison with the expected survival of these young patients. The ab- sence of many common toxic effects of bevacizumab in our patients may have reflected their relative youth, the lack of other medical conditions, and the small number of patients who were treated.

Our results show an interesting biologic effect of bevacizumab treatment on vestibular schwannomas associated with neurofibromatosis type 2 . However, we treated only patients with neurofibromatosis type 2 who were at imminent risk for severe neurologic consequences and not those with less advanced symptoms or patients with sporadic vestibular schwannomas. Additional research will be necessary to determine the optimal drug regimen, duration, and adverse-effect profile for long-term anti-VEGF therapy for vestibular schwannomas associated with neurofibromatosis type 2 .

Supported by the Harvard Medical School Center for Neurofibromatosis and Allied Disorders; Neurofibromatosis, Inc., New England; the Children's Tumor Foundation; a grant (NF050202, to Dr. Plotkin) from the Neurofibromatosis Research Program of the Department of Defense; a Claflin Award (to Dr. di Tomaso); grants (P01NS024279, to Dr. Stemmer-Rachamimov; and P01CA80124, to Dr. Jain) from the National Institutes of Health; and grants from the Federal Share/National Cancer Institute Proton Beam Program (to Drs. di Tomaso and Jain).

Dr. Plotkin reports receiving consulting fees from Novartis and grant support from Pfizer; Dr. Sorensen, receiving grant support from AstraZeneca, Exelixis, Schering-Plough, Genentech, Novartis, Takeda/Millennium, and Siemens Medical Solutions and consulting fees from AstraZeneca, Bayer Healthcare, Genentech, Mitsubishi Pharma, Novartis, and Takeda/Millennium; and Dr. Jain, receiving consulting fees from AstraZeneca, Dyax, Enlight, and Millennium and lecture fees from Roche Pharmaceuticals and Pfizer, having an equity interest in Enlight and SynDevRx, and receiving grant support from AstraZeneca and Dyax. No other potential conflict of interest relevant to this article was reported.

We thank Marybeth Singh for her clinical care and Drs. Meiyun Wang, Poe-jou Chen, and Dominique Jennings for their work in analyzing the imaging data.

\section{REFERENCES}

1. Evans DG, Moran A, King A, Saeed S, Gurusinghe N, Ramsden R. Incidence of vestibular schwannoma and neurofibromatosis 2 in the North West of England over a 10-year period: higher incidence than previously thought. Otol Neurotol 2005;26:93-7.

2. Samii M, Gerganov V, Samii A. Improved preservation of hearing and facial nerve function in vestibular schwannoma surgery via the retrosigmoid approach in a series of 200 patients. J Neurosurg 2006; 105:527-35.

3. Flickinger JC, Kondziolka D, Niranjan A, Lunsford LD. Results of acoustic neuroma radiosurgery: an analysis of 5 years' experience using current methods. J Neurosurg 2001;94:1-6.

4. Brackmann DE, Fayad JN, Slattery WH III, et al. Early proactive management of vestibular schwannomas in neurofibromatosis type 2. Neurosurgery 2001;49:274-80. 5. Combs SE, Volk S, Schulz-Ertner D, Huber PE, Thilmann C, Debus J. Management of acoustic neuromas with fractionated stereotactic radiotherapy (FSRT): long-term results in 106 patients treated in a single institution. Int J Radiat Oncol Biol Phys 2005;63:75-81.

6. Samii M, Matthies C, Tatagiba $M$. Management of vestibular schwannomas (acoustic neuromas): auditory and facial nerve function after resection of 120 vestibular schwannomas in patients with neurofibromatosis 2. Neurosurgery 1997 ; 40:696-705.

7. Folkman J. Tumor angiogenesis: therapeutic implications. N Engl J Med 1971; 285:1182-6.

8. Carmeliet P, Jain RK. Angiogenesis in cancer and other diseases. Nature 2000; 407:249-57.

9. Nagy JA, Benjamin L, Zeng H, Dvorak AM, Dvorak HF. Vascular permeability, vascular hyperpermeability and angiogenesis. Angiogenesis 2008;11:109-19.

10. Brieger J, Bedavanija A, Lehr HA, Maurer J, Mann WJ. Expression of angio- 
genic growth factors in acoustic neurinoma. Acta Otolaryngol 2003;123:1040-5.

11. Cayé-Thomasen P, Baandrup L, Jacobsen GK, Thomsen J, Stangerup SE. Immunohistochemical demonstration of vascular endothelial growth factor in vestibular schwannomas correlates to tumor growth rate. Laryngoscope 2003;113:2129-34.

12. Cayé-Thomasen P, Werther K, Nalla A, et al. VEGF and VEGF receptor- 1 concentration in vestibular schwannoma homogenates correlates to tumor growth rate. Otol Neurotol 2005;26:98-101.

13. Saito K, Kato M, Susaki N, Nagatani T, Nagasaka T, Yoshida J. Expression of Ki-67 antigen and vascular endothelial growth factor in sporadic and neurofibromatosis type 2-associated schwannomas. Clin Neuropathol 2003;22:30-4.

14. Uesaka T, Shono T, Suzuki SO, et al. Expression of VEGF and its receptor genes in intracranial schwannomas. J Neurooncol 2007;83:259-66.

15. Takamiya Y, Brem H, Ojeifo J, Mineta T, Martuza RL. AGM-1470 inhibits the growth of human glioblastoma cells in vitro and in vivo. Neurosurgery 1994;34: 869-75.

16. Mulvihill JJ, Parry DM, Sherman JL, Pikus A, Kaiser-Kupfer MI, Eldridge R. NIH conference: neurofibromatosis 1 (Recklinghausen disease) and neurofibromatosis 2 (bilateral acoustic neurofibromatosis): an update. Ann Intern Med 1990;113:39-52.
17. Harris GJ, Plotkin SR, MacCollin M, et al. Three-dimensional volumetrics for tracking vestibular schwannoma growth in neurofibromatosis type II. Neurosurgery 2008;62:1314-9.

18. Batchelor TT, Sorensen AG, di Tomaso E, et al. AZD2171, a pan-VEGF receptor tyrosine kinase inhibitor, normalizes tumor vasculature and alleviates edema in glioblastoma patients. Cancer Cell 2007; 11:83-95.

19. Monsell EM. New and revised reporting guidelines from the Committee on Hearing and Equilibrium, American Academy of Otolaryngology-Head and Neck Surgery Foundation, Inc. Otolaryngol Head Neck Surg 1995;113:176-8.

20. Hirsh IJ, Davis H, Silverman SR, Reynolds EG, Eldert E, Benson RW. Development of materials for speech audiometry. J Speech Hear Disord 1952;17:321-37. 21. Plotkin SR, Halpin C, Blakely JO, et al. Suggested response criteria for phase II antitumor drug studies for neurofibromatosis type 2 related vestibular schwannoma. J Neurooncol 2009;93:61-77.

22. Babovic-Vuksanovic D, Ballman K, Michels V, et al. Phase II trial of pirfenidone in adults with neurofibromatosis type 1. Neurology 2006;67:1860-2.

23. Babovic-Vuksanovic D, Widemann BC, Dombi E, et al. Phase I trial of pirfenidone in children with neurofibromatosis 1 and plexiform neurofibromas. Pediatr Neurol 2007;36:293-300.
24. Thornton AR, Raffin MJ. Speech-discrimination scores modeled as a binomial variable. J Speech Hear Res 1978;21:50718.

25. Halpin C, Rauch SD. Using audiometric thresholds and word recognition in a treatment study. Otol Neurotol 2006;27: 110-6.

26. Plotkin SR, Singh MA, O'Donnell CC, Harris GJ, McClatchey AI, Halpin C. Audiologic and radiographic response of NF2related vestibular schwannoma to erlotinib therapy. Nat Clin Pract Oncol 2008;5:48791.

27. Caye-Thomasen P, Dethloff T, Hansen $S$, Stangerup SE, Thomsen J. Hearing in patients with intracanalicular vestibular schwannomas. Audiol Neurootol 2007;12: 1-12.

28. Jain RK. Normalization of tumor vasculature: an emerging concept in antiangiogenic therapy. Science 2005;307:5862.

29. Kamoun WS, Ley CD, Farrar CT, et al. Edema control by cediranib, a vascular endothelial growth factor receptor-targeted kinase inhibitor, prolongs survival despite persistent brain tumor growth in mice. J Clin Oncol 2009;27:2542-52. 30. Fine HA, Figg WD, Jaeckle K, et al. Phase II trial of the antiangiogenic agent thalidomide in patients with recurrent high-grade gliomas. J Clin Oncol 2000;18: 708-15.

Copyright (c) 2009 Massachusetts Medical Society.

RECEIVE IMMEDIATE NOTIFICATION WHEN

A JOURNAL ARTICLE IS RELEASED EARLY

To be notified when an article is released early on the Web and to receive the table of contents of the Journal by e-mail every Wednesday evening, sign up through our Web site at NEJM.org. 